\title{
Axonal buckling following stretch injury
}

\author{
Georgina Lang, Sarah Waters, Dominic Vella, Alain Goriely, \\ Mathematical Institute, University of Oxford \\ E-mail: goriely@maths.ox.ac.uk \\ Corresponding author: A.G.
}

October 7, 2016

\begin{abstract}
Diffuse brain injury is caused by rapid rotation of the head, and causes strain injury to tissue throughout the brain. Following strain injury axons exhibit delayed recovery, showing regional buckling behavior immediately after stretch and returning to their original appearance over an extended period of time. This axonal buckling is hypothesized to occur as a result of localized stretching within the axon: Rapid strain causes mechanical damage to microtubules, increasing the effective length of axons. This damage is repaired gradually returning the axon to its initial length.

Here, we test the hypothesis that localized stretching is a possible explanation for the regional buckling behavior. An elongated region of axon is modeled as an Euler beam on an elastic foundation, where the foundation represents the surrounding brain tissue, which consists of glial cells and extracellular matrix. After stretch the elastic foundation returns immediately to its pre-stretch length, while the axon is initially elongated and returns to its original length over a longer period of time. The model exhibits solutions similar to those observed experimentally in post-stretch axons, with undulations that have a similar wavelength and amplitude.
\end{abstract}

\section{Introduction}

When axons are damaged by stretch injury due to tissue elongation, an interesting sequence of events occurs: Regions of the axon exhibit buckling immediately after injury, then the axon returns to its original appearance over a longer period of time. Review of the experimental literature suggests that this behavior is caused by localized stretching of regions of the axon, due to mechanical damage caused to the cytoskeleton within the axon. In this work we test whether localized stretching is a feasible mechanism to explain the observed behavior. To answer this question, we model an axon and the surrounding tissue as a beam on an elastic foundation.

\subsection{Axon mechanics}

The brain is the 'control centre' for the body and is a highly complex organ made from soft tissue. During everyday activity the brain is protected from damage by the skull and brain tissue is flexible 
enough to withstand the low forces that are applied from normal movement or light impact, such as running and jumping. However, extreme forces or impacts can lead to brain damage.

Brain tissue is very soft [20] and composed primarily of two cell types: neurons, which carry out information processing and signalling, and glial cells which are thought to carry out several support roles including providing electrical insulation to neurons, regulating extra-cellular ionic conditions, and offering structural support [31]. As illustrated in Figure 1, a typical neuron has a cell body, several processes called dendrites (which receive information from other neurons), and one axon (which transmits information to other neurons through connections with their dendrites). Since axons may be several centimetres long, they require both mechanical stability to prevent them from breaking under small forces, and efficient transport mechanisms to enable transport of nutrients and organelles within the cell. The cytoskeleton of a neuron extends through the cell body, axon and dendrites, offering structural support and facilitating intracellular transport [36]. Three protein polymers make up the cytoskeleton; microtubules, neurofilaments, and microfilaments. Microtubules are the stiffest component of the cytoskeleton [35], and maintain axonal shape by resisting compression [15]. They also have a role in intracellular transport, as motor proteins can 'walk' along the microtubules to transport components (such as mitochondria and vesicles) from one part of the cell to another [10]. Neurofilaments are the most abundant abundant cytoskeletal element in adult vertebrate neurons, and the core of an axon is made up of microtubules and neurofilaments, connected by crosslinking proteins $[12,22]$. Interestingly, some invertebrates such as Drosophila do not have neurofilaments [37] and neurofilaments are relatively rare in embryonic neurons (Yuan et al., 2009). Microfilaments, or actin filaments, are arranged in a layer just under the cell membrane and also exist throughout the cytoskeleton [18]. They are involved in axon growth at the growth cone, and also enable intracellular transport by acting as tracks for motor proteins [5].

Viewed as a mechanical structure, the entire axon can be modeled as a viscoelastic fluid that actively generate forces, and elongate and shorten as the result of stretching [7,34]. Equivalently they can be modeled as a morphoelastic rod [29] as discussed in [36]. However, in the first instance, when trying to understand the role of elastic forces in the buckling of axons, we can neglect internal viscous re-organization and growth. In that case, the initial response of an axon can be understood as a elastic rod on a foundation. Interestingly, finite-element modeling based on the interactions of microtubules also show buckling instability [43]. On longer time scales, there is an internal re-organization. This remodeling process can be modeled as a change in the effective length for the elastic problem.

\subsection{Axonal injury}

Traumatic brain injury is a general term describing damage or injury caused to the brain by an external mechanical force. There are two types of traumatic brain injury: focal and diffuse. Focal injury is caused by a direct blow to the head, and results in damage of brain tissue close to the site of impact. Symptoms can include accumulations of blood within the brain tissue (haematoma), bleeding into the subarachnoid space (subarachnoid haemorrhage) and bruising (contusions) [45, 24]. In contrast, diffuse brain injury is caused when the brain undergoes rapid acceleration, for example in a car crash [40]. When the resulting force is applied to the brain the white matter can deform, resulting in axons being stretched and damaged [21].

The strain rate is important: If axons are stretched over a period of several minutes they are able to withstand strains of over $100 \%$ and return to their original configuration with no evidence 


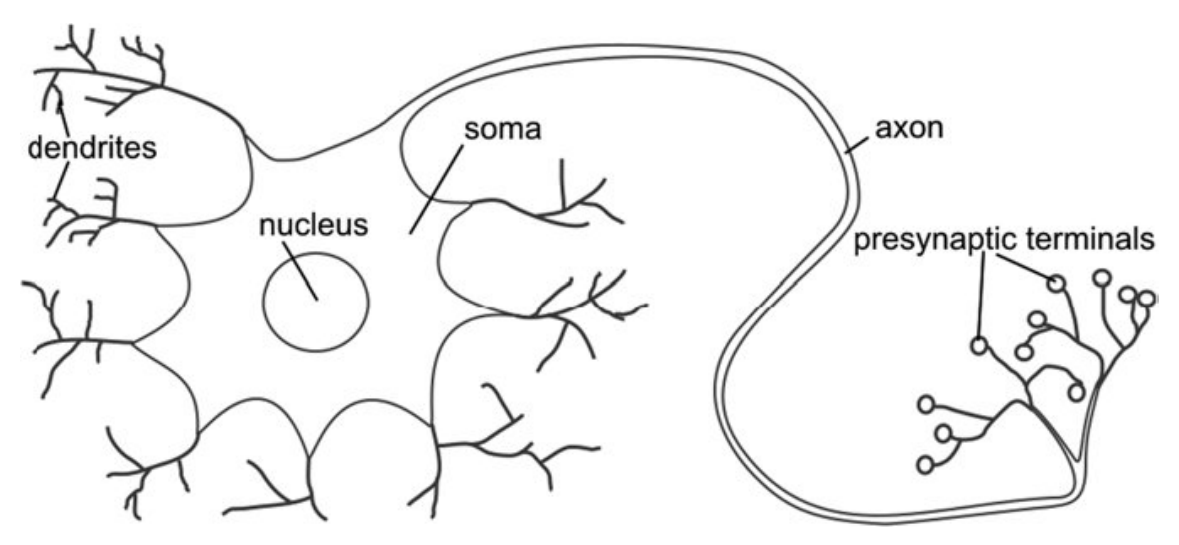

Figure 1: Schematic illustration showing the structure of a typical neuron (From [17])

of damage [44]; indeed low strain rates can stimulate axon growth [9, 42]. However, axons are damaged when rapidly stretched over a typical timescale of milliseconds. Accidents that lead to diffuse brain injury, such as car crashes, falls and assaults, all involve large strain rates being applied to the brain leading to this dynamic stretch injury.

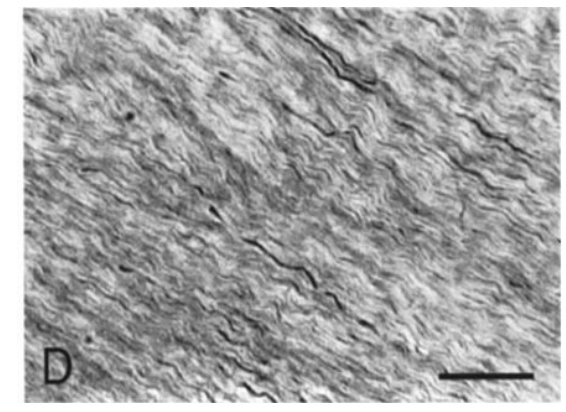

(A) Healthy tissue

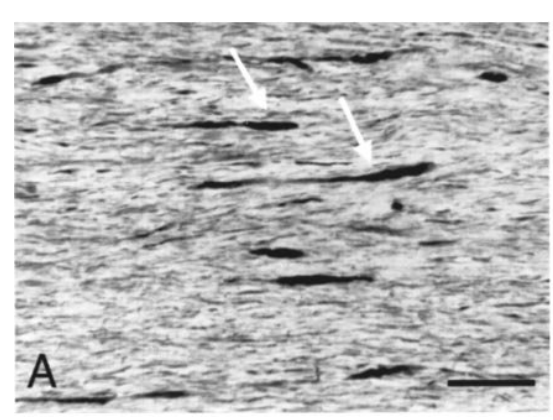

(B) Damaged tissue

Figure 2: Comparison of healthy and stretch damaged brain tissue, taken from an optic nerve

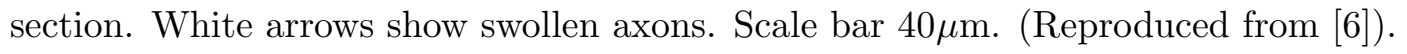

Following dynamic stretch injury axons may become broken and swollen [6]. This diffuse brain injury may be identified in tissue by regions of broken and swollen axons throughout the white matter (Figure 2). In primary axotomy, axons break immediately when strain is applied. However, in vitro experiments have shown that axons have good tolerance to rapid strain, and will not undergo primary axotomy when strains of up to $65 \%$ are applied sufficiently slowly [41].

\subsection{Secondary axonal damage}

The majority of axons become disconnected as a result of secondary axotomy, in which a sequence of mechanical and chemical changes result in disconnection some time after the strain is applied. After this stretch, unbroken axons show regions of delayed recovery: they exhibit buckling behavior immediately after the stretch is released then recover to a normal appearance within about 45 minutes (Figure 3). As shown experimentally by Smith et al. [41], after a longer period of time 


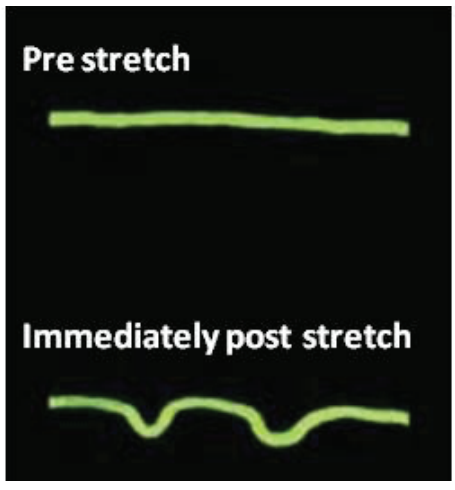

Initial event

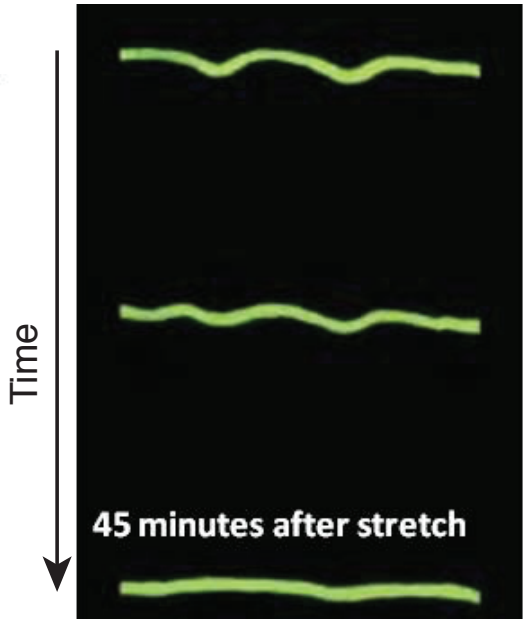

Relaxation

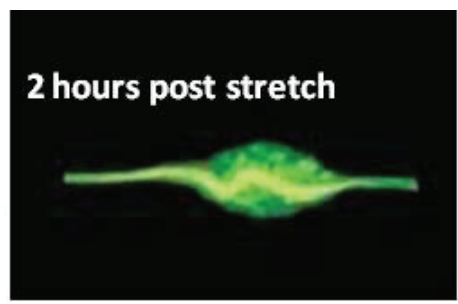

Damage

Figure 3: Demonstration of the delayed elastic behavior of an axon after dynamic stretch injury, illustrated by fluorescence microscopy. Adapted from [40].

(from 1 hour onwards) the axons can become swollen at the sites that originally showed buckling behavior, and eventually disconnect [40, 41].

Axons are brittle under rapid loading but elastic under slow loading [23]. The model of 'sliding filaments' of Chetta et al. [12] proposed that when a force is applied, crosslinks between microtubules break so that the microtubules can slide over each other. The crosslinks then reform once the material is at rest. As there is no damage to individual microtubules, transport mechanisms within the cell are not disrupted.

When exposed to dynamic stretch, it is thought that the microtubules connections are mechanically damaged [44]. Once the stretch is released, microtubules fail to realign properly, creating blocks that prevent the axons returning to their pre-stretch length, and resulting in undulations. After trauma, as the result of the forces acting on the system, microtubules then buckle and slide back together, allowing the shape of the the axons to appear to return to normal. However since microtubules are critical to transport within a cell, transport mechanisms with buckled microtubules [39] are disrupted which cause long-term problems.

Experiments in which single axons are stretched have showed that buckled regions will often exhibit swelling some time afterwards, subsequently causing secondary axotomy [41, 40]. The swellings appear to contain a central core of neurofilaments, which can be straight or undulated, surrounded by less dense accumulation of neurofilaments. The mechanism of axonal swelling and potential subsequent axotomy is a complicated process. It is thought to involve mechanical factors due to mechanical damage to cell membranes and microtubules, and chemical factors triggered by ionic changes in damaged regions [11].

The chemical changes that lead to axon swelling and secondary axotomy are very complex; several interacting processes are responsible for the changes that take place [16]. An influx of calcium is believed to be the chemical trigger leading to secondary axotomy: Mechanical damage to the cell membrane [3] and channel proteins [48] allows abnormal amounts of calcium to enter the cell. Experiments have shown abnormally high calcium concentrations within axons following 
traumatic injury [48]. Furthermore, high levels of calcium within the cell stimulate the release of calcium-dependent proteases [28], which break down microtubules and damage neurofilaments in the cytoskeleton. Damage to the cytoskeleton disrupts axonal transport, leading to accumulation of transport proteins and swelling [11]. High calcium concentrations can also damage mitochondria. The cell membrane of damaged mitochondria releases cytochrome-c [3], a protein that triggers apoptosis within the cell. After secondary axotomy has occurred the disconnected region of the axon is dissolved by Wallarian degradation and the neuron will either die or the axon will regenerate: axons within the peripheral nervous system may regenerate whilst those in the central nervous system may not [47]. An understanding of the internal damage of axons seem to be crucial to address problems related to tau-pathologies and various forms of dementia $[46,1]$.

\section{Problem set up}

\subsection{Model description}

The aim of this work is to test the hypothesis that localized internal stretching of axons is responsible for the buckling behavior observed following stretch injury. The elongated region of an axon is modeled as a planar Euler beam on an elastic foundation representing the brain tissue. A Winkler foundation is used to model the elastic foundation, which supposes that the force applied to the beam by the foundation is proportional to the transverse displacement of the beam.

The hypothesis of localized stretching is tested by assuming that after dynamic stretch the elastic foundation immediately returns to its original length, whilst the beam returns to its original length over an extended period of time. Immediately after stretch the elongated beam is longer than the foundation and therefore subject to compressive forces. A diagram of the model is shown in Figure 4. The balance between the resistance of the beam to bending and the elastic response of the foundation will determine the wavelength of the buckling.

\subsection{Assumptions}

In this simplified model, an axon is modeled as a one-dimensional beam, which may only be displaced transversely. It is assumed that the beam undergoes only small displacements and uniform elongation. We further assume that the foundation is homogeneous along the beam, and that contact is never broken between the beam and foundation. Since we assume that the elongation is confined within a given region of typical size $2 L_{0}$, there is no displacement outside the domain and we use clamped boundary conditions.

\subsection{Governing equations}

The transverse displacement of the beam is described by $w(x)$ on the domain $-L_{0}<x<L_{0}$. The solution will be given by the function $w(x)$ that minimizes the energy within the beam, subject to the constraint that the length of the beam, $L(t)$, is a prescribed function of time. Mathematically this is equivalent to minimizing the function

$$
\text { Energy }=\overbrace{\frac{B}{2} \int_{-L_{0}}^{L_{0}} \frac{w^{\prime \prime 2}}{1+w^{\prime 2}} \mathrm{~d} x}^{\text {bending energy }}+\overbrace{\frac{k}{2} \int_{-L_{0}}^{L_{0}} w^{2} \mathrm{~d} x}^{\text {foundation energy }},
$$




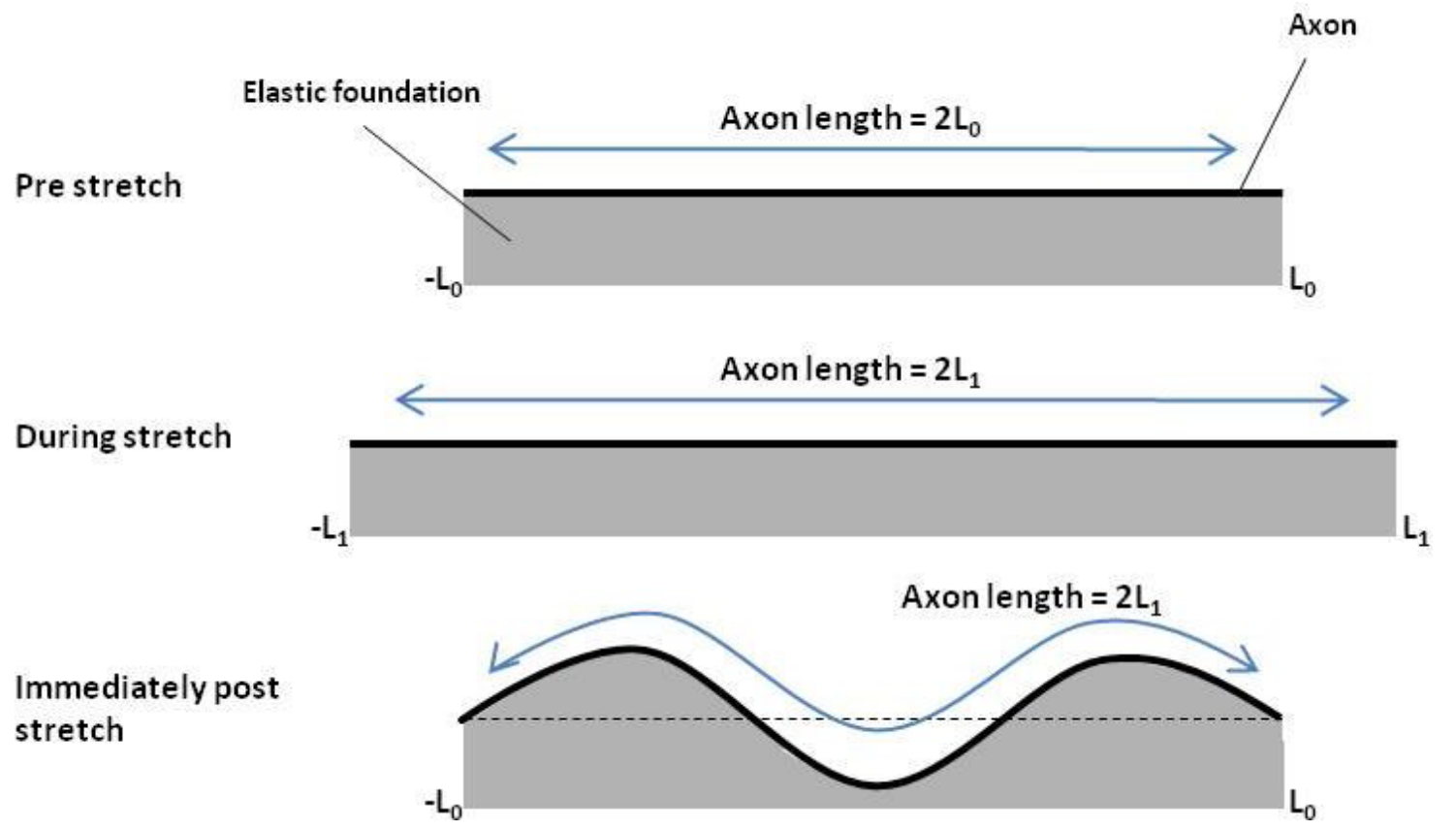

Figure 4: Diagram illustrating the Euler beam model on an elastic foundation

subject to the constraint

$$
2 L(t)=\int_{-L_{0}}^{L_{0}}\left(1+w^{\prime 2}\right)^{\frac{1}{2}} \mathrm{~d} x,
$$

where' denotes differentiation with respect to $x$. Here, $B$ (units $\mathrm{Nm}^{2}$ ) is the bending stiffness of the beam and $k$ (units $\mathrm{Nm}^{-2}$ ) is the elasticity of the foundation. We will discuss estimates for these parameters in Section 3. Introducing a Lagrange multiplier, $P$, gives the energy functional

$$
J[w(x)]=\int_{-L_{0}}^{L_{0}} \mathcal{F}\left[w, w^{\prime}, w^{\prime \prime}\right] \mathrm{d} x .
$$

where

$$
\mathcal{F}\left[w, w^{\prime}, w^{\prime \prime}\right]=\frac{B}{2} \frac{w^{\prime \prime 2}}{1+w^{\prime 2}}+\frac{k}{2} w^{2}-P\left(\left(1+w^{\prime 2}\right)^{\frac{1}{2}}-\frac{L}{L_{0}}\right) .
$$

The integrand is linearized by the assumption that the beam undergoes only small displacements, and the Euler-Lagrange equation for the problem reads

$$
\frac{\partial \mathcal{F}}{\partial w}-\frac{\mathrm{d}}{\mathrm{d} x} \frac{\partial \mathcal{F}}{\partial w^{\prime}}+\frac{\mathrm{d}^{2}}{\mathrm{~d} x^{2}} \frac{\partial \mathcal{F}}{\partial w^{\prime \prime}}=0
$$

Substituting $\mathcal{F}$ into the Euler-Lagrange equation gives:

$$
B \frac{\mathrm{d}^{4} w}{\mathrm{~d} x^{4}}+P \frac{\mathrm{d}^{2} w}{\mathrm{~d} x^{2}}+k w=0 .
$$

This equation can also be obtained as a reduction of the Kirchhoff equation to small planar transverse motion [30] or from a general description of Kirchhoff rods that can grow while attached to 
a support $[29,26]$. The force $P$, which was introduced as a Lagrange multiplier, can equivalently be thought of as the compressive force acting along the beam.

We have not considered any dynamical effect so far. Indeed, we are primarily interested in the quasi-static equilibrium states of the rod but not the dynamics associated to the buckling to these modes. However, we are interested in the evolution of the rod after a remodeling time ( 5 to 45 minutes) much longer than the typical viscous time scales (of the order of seconds or smaller). Denoting the extended length immediately after stretch by $L_{1}$, and allowing the length of the beam to decay over a characteristic time scale $\alpha$, the length of the beam at any time is prescribed by,

$$
L(t)=L_{0}+\left(L_{1}-L_{0}\right) e^{-t / \alpha} .
$$

This particular form for the relaxation can be understood by recalling that the axon is a viscoelastic material, where for which the typical relaxation scale is given as a ratio of internal viscosity to Young's modulus. When the axon is stretched, the elastic elements are stretched. Over time the stress in these elements relaxes and they return to their rest length. The rate at which this process occurs is determined by the ratio of the viscosity over the Young's Modulus of the axon.

To close these equations, a clamped boundary condition is imposed at both ends of the domain,

$$
w\left( \pm L_{0}\right)=0, \quad \frac{d w}{d x}\left( \pm L_{0}\right)=0 .
$$

Equations (6) to (8), together with the length constraint (2) are the full equations for the system.

\subsection{Non-dimensionalization}

Non-dimensionalising lengths with $L_{0}$ such that $w=L_{0} w^{*}$ and $x=L_{0} x^{*}$ and time with $\alpha$ so that $t=\alpha t^{*}$ gives the final system (dropping the stars),

$$
\frac{\mathrm{d}^{4} w}{\mathrm{~d} x^{4}}+\lambda \frac{\mathrm{d}^{2} w}{\mathrm{~d} x^{2}}+\beta w=0
$$

where

$$
\lambda=\frac{P L_{0}^{2}}{B}, \quad \beta=\frac{k L_{0}^{4}}{B},
$$

are dimensionless parameters. Linearizing equation (2) gives,

$$
2(\hat{L}(t)-1)=\int_{-1}^{1} \frac{1}{2}\left(\frac{\mathrm{d} w}{\mathrm{~d} x}\right)^{2} \mathrm{~d} x
$$

where $\hat{L}(t)=L(t) / L_{0}, \hat{L}_{1}=L_{1} / L_{0}$, and $\hat{L}(t)$ is the prescribed function of time,

$$
\hat{L}(t)=1+\left(\hat{L}_{1}-1\right) e^{-t} .
$$

The dimensionless clamped boundary conditions are

$$
w( \pm 1)=0, \quad \frac{\mathrm{d} w}{\mathrm{~d} x}( \pm 1)=0 .
$$

Equations (9) to (13) comprise the full system, and constitute a well defined problem. The only parameters in the system are the dimensionless parameters $\beta$ and $\lambda$ : here $\beta$ is a control parameter that can be interpreted as the length of the beam $L_{0}$ in terms of the characteristic buckling wavelength $\sim(\beta / k)^{1 / 2}$; conversely $\lambda$ is an unknown specified by the constraint (11). 


\section{Parameter analysis}

As with many physiological problems, obtaining precise parameter values can be a significant challenge. The properties of brain tissue vary with species, region, age, and even gender [17]; furthermore, experimental results are often inconsistent due to different methodologies, subjects, and experimental conditions [20, 14].

\subsection{Axon dimensions}

The length and radius of an individual axon is variable, and depends upon the species and age of the subject, as well as the location of the axon in the nervous system. The radius of an axon can range from $0.5 \mathrm{~mm}$ for the giant squid axon [13] down to fractions of microns. Here, we are primarily interested in understanding the behavior observed experimentally by Smith et al.[41], for which a culture of human cells was used. Smith et al. measured the diameter of axons in their culture to be in the range of $0.5 \mu \mathrm{m} \leq 2 r_{0} \leq 1.5 \mu \mathrm{m}$; we therefore take $r_{0}=0.5 \mu \mathrm{m}$ as a typical axon radius. This value is consistent with values reported for other mammals: axon diameters ranging from $0.1-2.5 \mu \mathrm{m}$ have been reported for monkey optic nerve neurons [38], $1-2.5 \mu \mathrm{m}$ for myelinated axons in the spinal cord of cats, cows and rats [27], and up to $4 \mu \mathrm{m}$ for porcine optic nerves [4].

The characteristic length of the region in which buckling occurs can be obtained from images of post-stretch axons published by Tang-Schomer et al. [44] and Smith et al. [41]. In each case, it is observed that the buckled region has length in the range $10 \mu \mathrm{m}$ to $20 \mu \mathrm{m}$ and so we shall consider the foundation length to lie within this range. Immediately after stretch, the overall length of axons has been reported to increase by up to $8 \%$, however this does not reflect the localized length increase within buckled regions since stretching is heterogeneous along the axon. Buckled regions may undergo a length increase of up to $60 \%$ [41], and therefore the elongated axon length $L_{1}$ is taken to be $60 \%$ greater than $L_{0}$.

It is consistently reported that axons return to the pre-stretch length over a period of approximately 45 minutes, and that the greatest rate of return to original length occurs within the first five minutes following stretch injury $[40,44]$. Therefore $\alpha$, the timescale for the recovery to original length, is taken as $\alpha=300 \mathrm{~s}$.

\subsection{Evaluating bending stiffness of an axon and elasticity of the foundation}

The key dimensionless parameter in the problem $\beta$, given in (10), depends on the axon bending stiffness, $B$, the foundation length, $2 L_{0}$, and the elasticity of the foundation, $k$. We therefore turn to the estimation of these parameters.

\subsubsection{Bending stiffness}

We consider two different ways to estimate the bending stiffness of an axon, either directly from the radius (modelling the axon as a homogeneous elastic rod) or by taking into account its internal structure.

For rods made of an isotropic, homogeneous, and linearly elastic material, the bending stiffness $B$ is the product of Young's modulus $E$ and second moment of area $I$, i.e., $B=E I$. The second moment of area for a cylindrical beam with radius $r_{0}$ is $I=p r_{0}^{4} / 4$, Therefore in order to obtain the second moment of area, only the radius of a typical axon is required. Using $r_{0}=0.5 \mu \mathrm{m}$ 
gives $I=4.9 \times 10^{-26} \mathrm{~m}^{4}$. Bernal et al. [7] experimentally measured the Young's modulus by of an individual axon to be $12 \mathrm{kPa}$. This was done by measuring the extensional response of axons, essentially measuring the product $\pi E r_{0}^{2}$ directly. Independent work obtained a value of $9 \mathrm{kPa}$ by nano-indentation [35]. These results are of the same order of magnitude, and so we take $E \approx 10 \mathrm{kPa}$, giving $B=4.9 \times 10^{-22} \mathrm{Nm}^{2}$. In Appendix $\mathrm{B}$, we compute an upper bound for the bending stiffness of an axon by considering the constituent parts of the cytoskeleton. This strict upper bound assumes internal rigid structures and leads to an estimate of $B \approx 6 \times 10^{-19} \mathrm{Nm}^{2}$, three order of magnitude larger than our previous estimate.

\subsubsection{Foundation stiffness}

It is difficult to measure the elasticity of the glial matter and extracellular matrix surrounding an axon [32]. Given an estimate for the elastic modulus of the material, $G$, the stiffness of the surrounding foundation may then be estimated assuming that the surrounding medium is homogeneous, incompressible and isotropic: in particular, the elasticity of the foundation is given by [8] $k=4 \pi G / \log \left(L_{\text {buck }} / r_{0}\right)$, where $L_{\text {buck }}$ is the characteristic length scale for buckling and $r_{0}$ is the rod radius. The problem with this estimate is that it depends on a quantity $L_{\text {buck }}$ that is itself the main unknown. It is therefore not suitable for modeling. Instead, we combine experimental measurements and an theoretical estimates for the growth velocity of an axon under tensional force to obtain an estimate for $\beta$.

\subsection{Experimental determination of $\beta$}

The main observation of O'Toole et al. [33] is that when an axon of length $L(t)$ is pulled with force $F_{0}$, the force profile along the axon is given by

$$
F(x)=F_{0} \frac{\cosh (x / a)}{\cosh (L(t) / a)},
$$

where $a$ is a typical length scale that can be measured from the length profile as a function of time and estimated experimentally at $a \approx 3.4 \times 10^{-5} \mathrm{~m}$. The problem of a pulled axon on an elastic substrate can be modeled as a growing elastic structure with an adhesive force attaching it to the substrate [29]. If the adhesive force is modeled as a constant multiple of the displacement of the axon, and it is assumed that the foundation has an isotropic response to displacement, the constant of proportionality is $k$. The axon stretches according to Hooke's Law, so that the strain acting on the axon is proportional to the stress experienced. Then the force profile across the axon is given by (14) with

$$
a=\sqrt{\frac{E A}{k}},
$$

where $A=\pi r_{0}^{2}$ is the cross-sectional area of the axon. Using the above of $a$ to eliminate $E / k$ from $\beta$, we find

$$
\beta=\frac{k L_{0}^{4}}{B}=\frac{4 L_{0}^{4}}{r_{0}^{2} a^{2}}
$$

With the measurement of $a$ from [33], we find that $\beta$ is dependent only upon the characteristic length scale $L_{0}$ and axon radius $r_{0}$. Indeed, using $a=3.4 \times 10^{-5} \mathrm{~m}$ and $r_{0}=0.5 \times 10^{-6} \mathrm{~m}$, gives $\beta=1.4 \times 10^{22} L_{0}^{4}$. This value for $\beta$ shall be used when solving the model. Note that this 
estimate relies on the underlying assumption that the typical relaxation times for the internal stress relaxation in the axons and the relaxation time of the substrate attachment are similar.

\section{Solution}

Since the equation for the deflection is linear, the solution of the system (9)-(13) can easily be obtained. General solutions to equation (9) have the form $w \sim e^{i k x}$ where $k$ satisfies the dispersion relation

$$
k^{4}-\lambda k^{2}+\beta=0 .
$$

Depending on the value of $\lambda^{2}-4 \beta$, there are three different forms for the general solution. If $\lambda^{2}-4 \beta=0$ then there are two real repeated roots for $k$, while if $\lambda^{2}-4 \beta<0$ then the four roots of $k$ take the form $k= \pm r \pm i \rho$. With clamped boundary conditions there are no non-trivial solutions to the problem for either of these cases, as shown in Appendix A. Thus only the case where $\lambda^{2}-4 \beta>0$ (corresponding to a buckled axon) is considered. Solutions take the form

$$
w=A \cos \left(k^{+} x\right)+B \cos \left(k^{-} x\right)+C \sin \left(k^{+} x\right)+D \sin \left(k^{-} x\right),
$$

where

$$
k^{-}=\sqrt{\frac{2 \lambda-\sqrt{\lambda^{2}-4 \beta}}{2}}, \quad k^{+}=\sqrt{\frac{2 \lambda+\sqrt{\lambda^{2}-4 \beta}}{2}} .
$$

The boundary conditions on $w( \pm 1)=0$ can be applied, and the resulting equations added and subtracted from each other to give

$$
\begin{aligned}
& A \cos \left(k^{+}\right)+B \cos \left(k^{-}\right)=0, \\
& C \sin \left(k^{+}\right)+D \sin \left(k^{-}\right)=0,
\end{aligned}
$$

and similarly the conditions $w^{\prime}( \pm 1)=0$ can be manipulated to give another pair of equations:

$$
\begin{aligned}
& A k^{+} \sin \left(k^{+}\right)+B k^{-} \sin \left(k^{-}\right)=0, \\
& C k^{+} \cos \left(k^{+}\right)+D k^{-} \cos \left(k^{-}\right)=0 .
\end{aligned}
$$

Solving these equations yields two possible solutions for $w(x)$. Either

$$
w(x)=A_{0}\left(\frac{\cos \left(k^{+} x\right)}{\cos \left(k^{+}\right)}-\frac{\cos \left(k^{-} x\right)}{\cos \left(k^{-}\right)}\right),
$$

if the dispersion relation $k^{+} \tan k^{+}=k^{-} \tan k^{-}$is satisfied, or

$$
w(x)=B_{0}\left(\frac{\sin \left(k^{+} x\right)}{\sin \left(k^{+}\right)}-\frac{\sin \left(k^{-} x\right)}{\sin \left(k^{-}\right)}\right),
$$

if $k^{+} \cot k^{+}=k^{-} \cot k^{-}$.

Since $k^{+}$and $k^{-}$are function of $\lambda, \lambda$ can be found from the solution to the dispersion relation. Note that for any $\beta$ each dispersion relation exhibits infinitely many solutions for $\lambda$. However, the solution that minimizes the energy of the system is the smallest value of $\lambda$ satisfying one or other of the dispersion relations, subject to the constraint $\lambda^{2}-4 \beta>0$. Thus the solution takes the form (24) or (25) depending upon which dispersion relation permits the smaller value for $\lambda$. 
A numerical solver is used to solve each of the dispersion relations and obtain values $k^{+}$, $k^{-}$, and $\lambda$. Once these have been found, the amplitude of the solution at any time is found by substituting the solution into the length constraint, given in equation (11). The system can then be redimensionalized to obtain the dimensional solution.

\section{Results}

\subsection{Behaviour immediately following stretch}

Through our rescaling, the value of $\beta$ is dependent on the fourth power of the unknown length, $L_{0}$; as a result, the shape of the beam is sensitively dependent on the value $L_{0}$. Figure 5 shows the results of our model for foundation lengths from $10 \mu \mathrm{m} \leq 2 L_{0} \leq 30 \mu \mathrm{m}$. As the length of the beam increases, the number of peaks and troughs increases. Physically, a larger value of $\beta$ means that deforming the foundation has become more 'expensive' in comparison to bending the beam and so, to minimize the energy of the system while accommodating the imposed compression, the beam must adopt a more convoluted, bent shape.

Comparing results of these simulations with published experimental results demonstrates qualitatively similar behavior. Tang-Schomer et al. [44] show images of a $15 \mu \mathrm{m}$ section of axon shortly following stretch injury. We note that the observed shape is qualitatively very similar to that predicted by our model (see Figure 5c), though we emphasize that the parameters here are not precisely known.
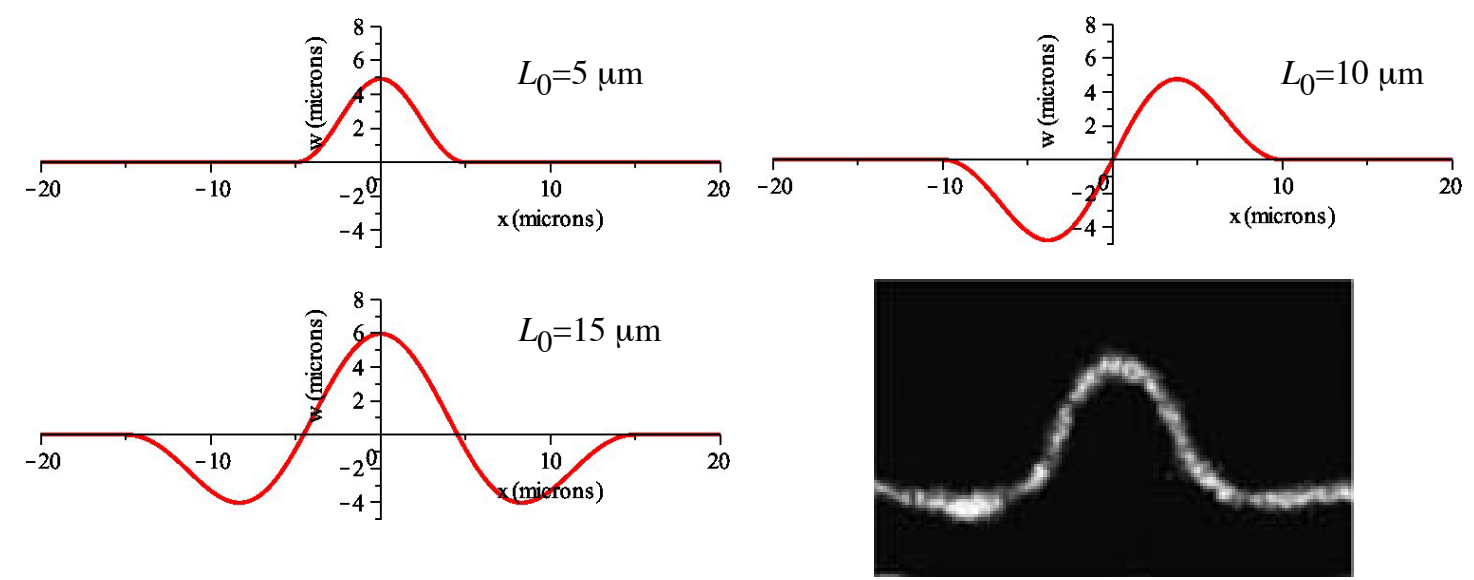

Figure 5: Solutions to the model at time $t=0$, for elongated regions of different lengths and Image of a buckled region of axon, shortly following stretch injury. Width of image is $15 \mu \mathrm{m}$ [44].

\subsection{Evolution of the axon after injury}

Following injury, an axon will return to its original appearance over a period of approximately 45 minutes. This can easily be modeled in our system by introducing the time-dependent axon length (12). Figure 6 demonstrates that our model also displays this behavior: the effect of reducing the axon length over time is to reduce the amplitude of the displacement. Indeed, returning to Figure 


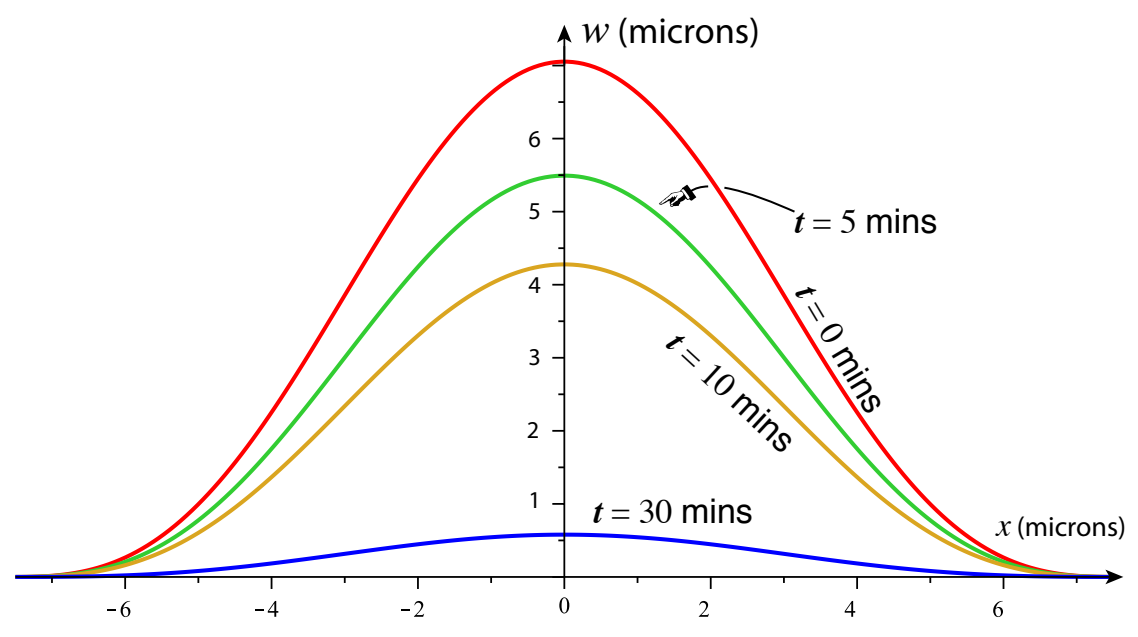

Figure 6: Results of the model for $L_{0}=7.5 \mu \mathrm{m}$, showing evolution of the solution over time.

3 , and considering a localized region close to one of the 'buckles', it can be seen that the behavior of the experiment is qualitatively similar to that predicted by the model.

\section{Discussion}

Our results support the idea that axon behavior after stretch is a consequence of buckling and localized elongation: initially, the elongated regions buckle due to elongation. On a longer time scale, the internal remodeling of the axon shortens the length and the amplitude of the buckle decreases, as observed experimentally [44]. It is therefore plausible that mechanical damage to microtubules causes localized lengthening of the axon. However, the model includes numerous simplifications. It is unlikely that a region of axon increases uniformly in length, and therefore more realistic behavior may be observed by considering heterogeneous elongation within the stretched region.

The elasticity of the foundation proved very difficult to evaluate, and it was assumed that the foundation was elastic, isotropic and homogeneous. Since the foundation surrounding an axon is made from glial cells and extracellular matrix, in reality these may also be affected by stretch injury so that the buckling may be caused by a combination of localized stretching of the axon and heterogeneous elastic response of the surrounding tissue. Furthermore, the bending stiffness of the axon is likely to be affected by stretch injury - the effects of a heterogeneous bending stiffness should also be considered. It would be a simple modelling exercise to consider a hierarchy of increasingly sophisticated models for the response of the foundation and the axon by including such effects as the nonlinear response of the foundation, a more accurate geometric model of the axon as well as heterogeneity, anisotropy and viscoelasticity. However, we do not expect any different qualitative behavior to be observed with such complications. The real bottleneck in our study has been the estimation of the biologically relevant parameters; as such the simple model presented here is a useful first step to motivate further experimental work. Recent numerical work on the buckling and torsional behavior of bundles of microtubules is particularly relevant here as it will 
allow to bridge between cellular and sub-cellular levels $[43,25]$. Despite the various simplifications and unknown parameters, the model compares favorably with experimental results and we conclude that theoretical results support the hypothesis that localized stretching is a plausible mechanism to explain the behavior of axons following stretch injury.

Acknowledgments: The research leading to these results has received funding from the European Research Council under the European Unions Horizon 2020 Programme / ERC Grant Agreement no. 637334 (DV).

\section{Appendices}

\section{A Solutions to the model for $\lambda^{2} \leq 4 \beta$}

This appendix shows that there are no non-trivial solutions to the system of equations (9)-(13) unless $\lambda^{2}>4 \beta$.

\section{A.1 $\lambda^{2}=4 \beta$}

Initially, we consider the case $\lambda^{2}=4 \beta$. Note that since $\beta$ is positive constant, this implies that $\lambda$ is non-zero and so the general solution to equation (9) is

$$
w=A \cos (k x)+B x \cos (k x)+C \sin (k x)+D x \sin (k x),
$$

where $k=\lambda / 2$. By assumption, the beam is clamped at the boundaries, so that the appropriate boundary conditions are those of (13). Applying the boundary condition $w( \pm 1)=0$ and adding and subtracting the equations obtained gives:

$$
\begin{aligned}
& A \cos (k)+D \sin (k)=0, \\
& B \cos (k)+C \sin (k)=0,
\end{aligned}
$$

while proceeding similarly with the boundary condition $w^{\prime}( \pm 1)=0$ we find another pair of equations:

$$
\begin{aligned}
& (D-A k) \sin (k)+D k \cos k=0, \\
& (C k+B) \cos (k)-B k \sin k=0 .
\end{aligned}
$$

Solving equations (27) and (29) gives that either $A=D=0$ or the dispersion relation $\sin k \cos k-$ $k=0$ is satisfied. Graphically it can be shown that the only solution to this dispersion relation is $k=0$, which is inconsistent with $k=\lambda / 2=\sqrt{\beta}>0$. We therefore conclude that $A=D=0$. Similarly, solving equations (28) and (30) gives that either $B=C=0$ or $\sin k \cos k+k=0$; the only solution to this equation is again $k=0$ and so $B=C=0$. As a result, no non-trivial solution exists that satisfies the clamped boundary conditions with $\lambda^{2}=4 \beta$.

\section{A.2 $\lambda^{2}<4 \beta$}

In the case $\lambda^{2}<4 \beta$ the general solution to equation (9) is given by:

$$
w=A \cos (r x) \sinh (\rho x)+B \cos (r x) \cosh (\rho x)+C \sin (r x) \sinh (\rho x)+D \sin (r x) \sinh (\rho x),
$$


where $k= \pm r \pm i \rho$ are the four solutions of the auxiliary equation $k^{4}-\lambda k^{2}+\beta=0$. Applying the boundary conditions at $w( \pm 1)$ and adding and subtracting the results gives:

$$
\begin{aligned}
& A \cos r \sinh \rho+D \sin r \cosh \rho=0, \\
& B \cos r \cosh \rho+C \sin r \sinh \rho=0 .
\end{aligned}
$$

Similarly imposing $w^{\prime}( \pm 1)=0$ and manipulating we find another pair of equations:

$$
\begin{aligned}
& 0=A(-r \sin r \sinh \rho+\rho \cos r \cosh \rho)+D(r \cos r \cosh \rho+\rho \sin r \sinh \rho), \\
& 0=B(-r \sin r \cosh \rho+\rho \cos r \sinh \rho)+C(r \cos r \sin \rho+\rho \sin r \cosh \rho) .
\end{aligned}
$$

Solving the equations (32) and (34) we obtain that either $A=D$ or the dispersion relationship $\cos r \sin r / r=\sinh \rho \cosh \rho / \rho$ holds. Graphically it can be shown that the only solution to the dispersion relation is $\rho=r=0$, contradicting our assumption that the root of the auxiliary equation is complex. Similarly solving (33) and (35) gives that either $B=C$ or $r$ and $\rho$ satisfy $\cos r \sin r / r=-\sinh \rho \cosh \rho / \rho$, which also has no non-trivial solutions. We therefore conclude that no non-trivial solutions exist that satisfy the clamped boundary conditions when $\lambda^{2}<4 \beta$.

\section{B Second moment of area for a circular arrangement of micro- tubules}

An upper bound, for the bending stiffness of an axon can be estimated by considering the constituent parts within the axon. Microtubules are the stiffest component of the cytoskeleton [35], and so we consider a structure consisting only of microtubules, whose Young's modulus and radius have been extensively characterized $[19,2]$. It is assumed that microtubules are arranged in a circular pattern located close to the perimeter of the axon, as shown in Figure 7(a). This assumption of arranging the microtubules around the outermost boundary of the axon and neglecting sliding between axons gives an upper bound for stiffness. If $E_{m}$ is the Young's modulus of microtubules and $I_{m}$ is the second moment of area for the arrangement of microtubules, then $B=E_{m} I_{m}$. For an arrangement of $N$ microtubules, each with radius $r_{m}$, and arranged at a radius $R$ from the centre of an axon, the second moment of area can be shown to be $\pi r_{m}^{2} R^{2} N / 2$.

The second moment of area for an arrangement of $N$ microtubules each with radius $r_{m}$ and positioned in a circular arrangement at a distance $R$ from the centre of an axon, as illustrated in Figure 7 (b) is given by $\pi r_{m}^{2} R^{2} N / 2$. First consider the moment of area $I$ of a single microtubule, with centre located at distance $a_{j}$ from the vertical axis. The second moment of area is given by,

$$
\begin{aligned}
I\left(a_{j}\right) & =\int_{D} z^{2} \mathrm{~d} z \mathrm{~d} y=\int_{-r_{m}}^{r_{m}} \int_{a-\sqrt{r_{m}^{2}-y^{2}}}^{a+\sqrt{r_{m}^{2}-y^{2}}} z^{2} \mathrm{~d} z \mathrm{~d} y \\
& =\frac{1}{3} \int_{-r_{m}}^{r_{m}}\left(a+\sqrt{r_{m}^{2}-y^{2}}\right)^{3}-\left(a-\sqrt{r_{m}^{2}-y^{2}}\right)^{3} \mathrm{~d} y \\
& =\frac{1}{3} \int_{-r_{m}}^{r_{m}} 8{\sqrt{r_{m}^{2}-y^{2}}}^{3}+6\left(a^{2}-\left(r_{m}^{2}-y^{2}\right)\right)\left(\sqrt{r_{m}^{2}-y^{2}}\right) \mathrm{d} y
\end{aligned}
$$



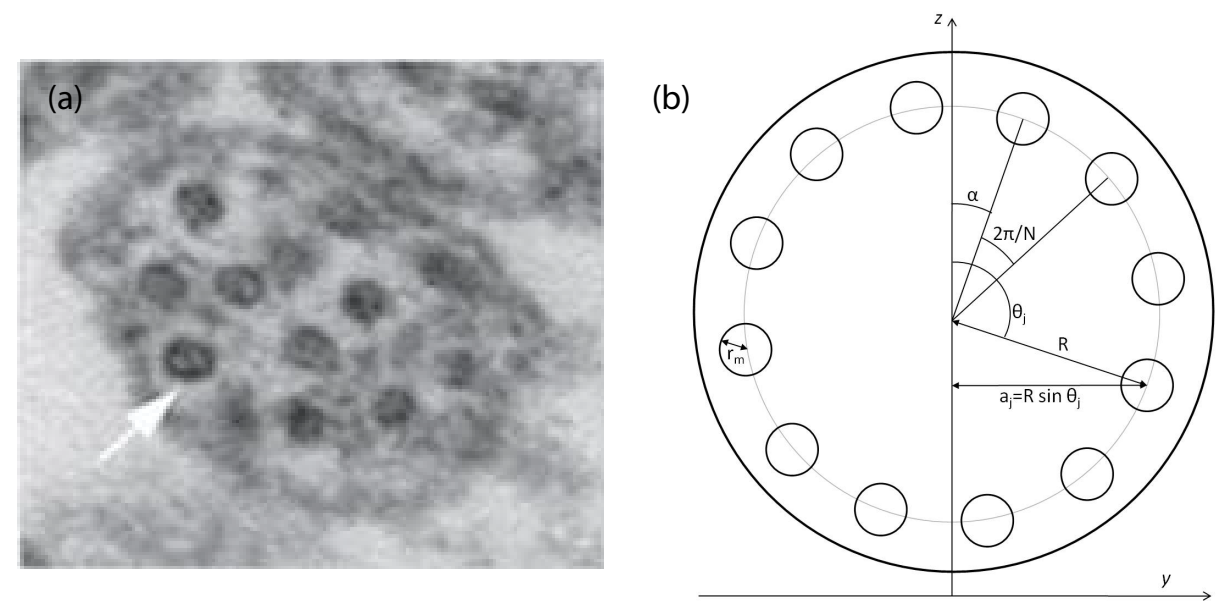

Figure 7: Cross section of an axon, showing the microtubules (arrow). Axon radius $=0.5 \mu \mathrm{m}$. Source: [44]. (b) Diagram illustrating the arrangement of $N=12$ microtubules which are used to calculate the second moment of area of an axon.

Parameterizing $y$ by $r_{m} \sin \theta$ for $\theta \in[-\pi / 2, \pi / 2]$. Then the integral simplifies to,

$$
\begin{aligned}
I\left(a_{j}\right) & =\frac{1}{3} \quad \int_{-\frac{\pi}{2}}^{\frac{\pi}{2}} 8 r_{m}^{4} \cos ^{4} \theta+6\left(a^{2}-r_{m}^{2} \cos ^{2} \theta\right) \mathrm{d} \theta \\
& =\frac{2}{3} \int_{-\frac{\pi}{2}}^{\frac{\pi}{2}} 2 r_{m}^{4}\left(\frac{3}{8}+\frac{1}{2} \cos 2 \theta+\frac{1}{8} \cos 4 \theta\right)+3 a_{j}^{2} r_{m}^{2}(1+\cos \theta) \mathrm{d} \theta, \\
& =\pi r_{m}^{2}\left(\frac{r_{m}^{2}}{4}+a_{j}^{2}\right) .
\end{aligned}
$$

Now considering a circular arrangement of $N$ microtubules, evenly spaced at radius $R$ from the centre of the axon. The distance from the vertical axis of each microtubules is given by $a_{j}=R \sin \theta_{j}$ where $\theta_{j}=\alpha+\frac{2 \pi(j-1)}{N}$. The second moment of area of the collection is given by:

$$
\begin{aligned}
I & =\sum_{j=i}^{N} I\left(a_{j}\right)=\sum_{j=i}^{N} \pi r_{m}^{2}\left(\frac{r_{m}^{2}}{4}+\left(R \sin \theta_{j}\right)^{2}\right), \\
& =\frac{N \pi r_{m}^{4}}{4}+\pi r_{m}^{2} R^{2} \sum_{j=i}^{N} \sin ^{2}\left(\alpha+(j-1) \frac{2 \pi}{N}\right) .
\end{aligned}
$$

Finally it remains to evaluate the sum, it can be shown that for all $N>2$ and all $\alpha$ the sum in the 
equation above is $N / 2$

$$
\begin{aligned}
\sum_{j=i}^{N} \sin ^{2}\left(\alpha+(j-1) \frac{2 \pi}{N}\right) & =\sum_{j=0}^{N-1}\left(\frac{1}{2 i}\right)^{2}\left(e^{\left(\alpha+\frac{2 \pi j}{N}\right) i}-e^{-\left(\alpha+\frac{2 \pi j}{N}\right) i}\right) \\
& =\frac{N}{2}-\frac{1}{4}\left(e^{2 \alpha i} \sum_{j=0}^{N-1} e^{\frac{4 \pi i}{N} j}+e^{-2 \alpha i} \sum_{j=0}^{N-1} e^{-\frac{4 \pi i}{N} j}\right) \\
& =\frac{N}{2}-\frac{1}{4}\left(e^{2 \alpha i}\left(\frac{1-e^{4 \pi i}}{1-e^{4 \pi i / N}}\right)+e^{-2 \alpha i}\left(\frac{1-e^{-4 \pi i}}{1-e^{-4 \pi i / N}}\right)\right)
\end{aligned}
$$

The numerators $1-e^{ \pm 4 \pi i}$ are always equal to zero, and the denominator $1-e^{ \pm 4 \pi i / N}$ is non zero unless $N=1$ or 2 . Thus for $N>2$,

$$
I=\frac{N \pi r_{m}^{4}}{4}+\pi r_{m}^{2} R^{2} \frac{N}{2}=\frac{\pi r_{m}^{2} N}{2}\left(\frac{r_{m}^{2}}{2}+R^{2}\right) \approx \frac{\pi r_{m}^{2} R^{2} N}{2}
$$

where an approximation is made since the radius of a microtubule $r_{m} \ll R$ - the radius at which the microtubules are arranged within the axon $R$.

The typical diameter of a microtubule $r_{m} \approx 25 \mathrm{~nm}[2]$ and, as previously, we take the radius of an axon $r_{0} \approx 0.5 \mu \mathrm{m}$. It is assumed that the microtubules are arranged close to the edge of the axon so that $R=0.4 \mu \mathrm{m}$, and assume that there are $N=12$ microtubules in a cross section. This number varies between axons but, as the bending stiffness varies only linearly with $N$, variations in $N$ will not have a great effect. Taking $E_{m} \approx 1.2 \mathrm{GPa}[19]$ we have,

$$
B=E_{m} I_{m}=E_{m} \times \frac{\pi r_{m}^{2} R^{2} N}{2} \approx 6 \times 10^{-19} \mathrm{Nm}^{2} .
$$

The cytoskeleton also consists of neurofilaments and microfilaments. Their overall contribution to the bending stiffness of axons are not fully understood. 


\section{References}

[1] Hossein Ahmadzadeh, Douglas H Smith, and Vivek B Shenoy. Mechanical effects of dynamic binding between tau proteins on microtubules during axonal injury. Biophysical journal, 109(11):2328-2337, 2015.

[2] B. Alberts. Molecular biology of the cell: Reference edition. Number v. 1 in Molecular Biology of the Cell: Reference Edition. Garland Science, 2008.

[3] Teuntje M. J. C. Andriessen, Bram Jacobs, and Pieter E. Vos. Clinical characteristics and pathophysiological mechanisms of focal and diffuse traumatic brain injury. Journal of Cellular and Molecular Medicine, 14(10):2381-2392, 2010.

[4] Yaniv Assaf, Tamar Blumenfeld-Katzir, Yossi Yovel, and Peter J. Basser. Axcaliber: A method for measuring axon diameter distribution from diffusion mri. Magnetic Resonance in Medicine, 59(6):1347-1354, 2008.

[5] PW Baas and A Karabay. Axonal Transport and the Neuronal Cytoskeleton. John Wiley \& Sons, Ltd, 2001.

[6] Allison C. Bain and David F. Meaney. Tissue-level thresholds for axonal damage in an experimental model of central nervous system white matter injury. Journal of Biomechanical Engineering, 122(6):615-622, 2000.

[7] Roberto Bernal, Pramod A. Pullarkat, and Francisco Melo. Mechanical properties of axons. Phys. Rev. Lett., 99(1):018301, Jul 2007.

[8] Clifford P. Brangwynne, Frederick C. MacKintosh, Sanjay Kumar, Nicholas A. Geisse, Jennifer Talbot, L. Mahadevan, Kevin K. Parker, Donald E. Ingber, and David A. Weitz. Microtubules can bear enhanced compressive loads in living cells because of lateral reinforcement. The Journal of Cell Biology, 173(5):733-741, 2006.

[9] Dennis Bray. Axonal growth in response to experimentally applied mechanical tension. Developmental biology, 102(2):379-389, 1984.

[10] Paul C Bressloff and Jay M Newby. Stochastic models of intracellular transport. Reviews of Modern Physics, 85(1):135, 2013.

[11] A. Büki and J. T. Povlishock. All roads lead to disconnection? Traumatic axonal injury revisited. Acta Neurochirurgica, 148:181-194, 2006.

[12] Joshua Chetta, Cecilia Kye, and Sameer B. Shah. Cytoskeletal dynamics in response to tensile loading of mammalian axons. Cytoskeleton, 67(10):650-665, 2010.

[13] Kenneth S. Cole and Howard J. Curtis. Electric impedance of the squid giant axon during activity. The Journal of General Physiology, 22(5):649-670, 1939.

[14] Rijk de Rooij and Ellen Kuhl. Constitutive modeling of brain tissue: Current perspectives. Appl. Mech. Rev., 68(1):010801, 2016. 
[15] TJ Dennerll, HC Joshi, VL Steel, RE Buxbaum, and SR Heidemann. Tension and compression in the cytoskeleton of pc-12 neurites. ii: Quantitative measurements. The Journal of cell biology, 107(2):665-674, 1988.

[16] Orsolya Farkas and John T. Povlishock. Cellular and subcellular change evoked by diffuse traumatic brain injury: a complex web of change extending far beyond focal damage. In John T. Weber and Andrew I.R. Maas, editors, Neurotrauma: New Insights into Pathology and Treatment, volume 161 of Progress in Brain Research, pages 43 - 59. Elsevier, 2007.

[17] Kristian Franze and Jochen Guck. The biophysics of neuronal growth. Reports on Progress in Physics, 73(9):094601, 2010.

[18] Archan Ganguly, Yong Tang, Lina Wang, Kelsey Ladt, Jonathan Loi, Bénédicte Dargent, Christophe Leterrier, and Subhojit Roy. A dynamic formin-dependent deep f-actin network in axons. The Journal of cell biology, 210(3):401-417, 2015.

[19] F Gittes, B Mickey, J Nettleton, and J Howard. Flexural rigidity of microtubules and actin filaments measured from thermal fluctuations in shape. The Journal of Cell Biology, 120(4):923934, 1993.

[20] A. Goriely, S. Budday, and E. Kuhl. Neuromechanics: From Neurons to Brain. Advances in Applied Mechanics, 48:79-139, 2015.

[21] A. Goriely, M. G. D. Geers, G. A. Holzapfel, J. Jayamohan, A. Jérusalem, S. Sivaloganathan, W. Squier, J. A. W. van Dommelen, S. L. Waters, and E. Kuhl. Mechanics of the brain: perspectives, challenges, and opportunities. Biomech. Model. Mechanobiol., 14:931, 2015.

[22] Nobutaka Hirokawa. Cross-linker system between neurofilaments, microtubules and membranous organelles in frog axons revealed by the quick-freeze, deep-etching method. The Journal of cell biology, 94(1):129-142, 1982.

[23] Maria A Holland, Kyle E Miller, and Ellen Kuhl. Emerging brain morphologies from axonal elongation. Ann. Biomed. Eng., 43(7):1640-1653, 2015.

[24] G. E. Lang, D. Vella, S. L. Waters, and A. Goriely. Propagation of damage in brain tissue: Coupling the mechanics of edema and oxygen delivery. Biomech. Model Mechanobiol., 14:11971216, 2015.

[25] Carole Lazarus, Mohammad Soheilypour, and Mohammad RK Mofrad. Torsional behavior of axonal microtubule bundles. Biophysical journal, 109(2):231-239, 2015.

[26] Th. Lessinnes, D. E. Moulton, and A. Goriely. Morphoelastic rods. Part II: Growing birods. J. Mech. Phys. Solids, 2015.

[27] Murray A. Matthews. An electron microscopic study of the relationship between axon diameter and the initiation of myelin production in the peripheral nervous system. The Anatomical Record, 161(3):337-351, 1968.

[28] William L. Maxwell, John T. Povlishock, and David L. Graham. A mechanistic analysis of nondisruptive axonal injury: A review. Journal of Neurotrauma, 14:419-440, 2007. 
[29] D E Moulton, T Lessinnes, and A Goriely. Morphoelastic rods Part I: A single growing elastic rod. J. Mech. Phys. Solids, 61(2):398-427, 2012.

[30] S. Neukirch, J. Frelat, A. Goriely, and C. Maurini. Vibrations of post-buckled rods: The singular inextensible limit. J. Sound Vib., 331(3):704-720, 2012.

[31] John Nolte. The Human Brain: An Introduction to Its Functional Anatomy. C.V. Mosby; 4th edition, 4th edition edition, August 1998.

[32] Stephen G O'Keeffe, Derek E Moulton, Sarah L Waters, and Alain Goriely. Growth-induced axial buckling of a slender elastic filament embedded in an isotropic elastic matrix. Int. J. Nonlinear Mech., 56:94-104, 2013.

[33] M. O'Toole, P. Lamoureux, and K. E. Miller. A physical model of axonal elongation: Force, viscosity, and adhesions govern the mode of outgrowth. Biophys. J., 94(7):2610-2620, 2008.

[34] Matthew O'Toole, Phillip Lamoureux, and Kyle E Miller. Measurement of subcellular force generation in neurons. Biophysical journal, 108(5):1027-1037, 2015.

[35] Hui Ouyang, E. Nauman, and Riyi Shi. Contribution of cytoskeletal elements to the mechanical property of axons. In Micro/Nano Symposium (UGIM), 2010 18th Biennial University/Government/Industry, pages 1-5, July 20102010.

[36] Pierre Recho, Antoine Jerusalem, and Alain Goriely. Growth, collapse, and stalling in a mechanical model for neurite motility. Phys. Rev. E, 93(3):032410, 2016.

[37] Melissa M Rolls. Neuronal polarity in drosophila: sorting out axons and dendrites. Developmental neurobiology, 71(6):419-429, 2011.

[38] R M Sanchez, G R Dunkelberger, and H A Quigley. The number and diameter distribution of axons in the monkey optic nerve. Investigative Ophthalmology \& Visual Science, 27(9):1342-50, 1986.

[39] Laura Schaedel, Karin John, Jérémie Gaillard, Maxence V Nachury, Laurent Blanchoin, and Manuel Théry. Microtubules self-repair in response to mechanical stress. Nature materials, 14(11):1156-1163, 2015.

[40] Douglas H. Smith and David F Meaney. Axonal damage in traumatic brain injury. The Neuroscientist, 6(6):483-495, 2000.

[41] Douglas H. Smith, John A. Wolf, Theresa A. Lusardi, Virginia M.-Y. Lee, and David F. Meaney. High tolerance and delayed elastic response of cultured axons to dynamic stretch injury. The Journal of Neuroscience, 19(11):4263-4269, 1999.

[42] Douglas H. Smith, John A. Wolf, and David F. Meaney. A new strategy to produce sustained growth of central nervous system axons: Continuous mechanical tension. Tissue Engineering, $7(2): 131-139,2001$.

[43] Mohammad Soheilypour, Mohaddeseh Peyro, Stephen J Peter, and Mohammad RK Mofrad. Buckling behavior of individual and bundled microtubules. Biophysical journal, 108(7):1718$1726,2015$. 
[44] Min D. Tang-Schomer, Ankur R. Patel, Peter W. Baas, and Douglas H. Smith. Mechanical breaking of microtubules in axons during dynamic stretch injury underlies delayed elasticity, microtubule disassembly, and axon degeneration. The FASEB Journal, 24(5):1401-1410, 2010.

[45] A.W. Unterberg, J. Stover, B. Kress, and K.L. Kiening. Edema and brain trauma. Neuroscience, 129(4):1019 - 1027, 2004. Brain Water Homeostasis.

[46] Henry van den Bedem and Ellen Kuhl. Tau-ism: The yin and yang of microtubule sliding, detachment, and rupture. Biophysical journal, 109(11):2215-2217, 2015.

[47] Mauricio E. Vargas and Ben A. Barres. Why is Wallerian degeneration in the CNS so slow? Annual Review of Neuroscience, 30(1):153-179, 2007.

[48] John A. Wolf, Peter K. Stys, Theresa Lusardi, David Meaney, and Douglas H. Smith. Traumatic axonal injury induces calcium influx modulated by tetrodotoxin-sensitive sodium channels. The Journal of Neuroscience, 21(6):1923-1930, 2001. 